celebration. His team is still analysing the data from the trial, and has not revealed how many bats were treated and released or how the controls fared. And even if this or the bacterial treatments are effective, they will be only short-term solutions. The fungus lingers on cave walls during the summer, and bats do not seem to develop immunity to it - so researchers would need to treat the animals every year to keep them from getting sick.

\section{STOPGAP SOLUTIONS}

Long-lasting solutions remain elusive. Some scientists hope to develop a vaccine, but have yet to work out how to trigger the animals' immune systems to destroy the pathogen, says Ken Field, an ecoimmunologist at Bucknell University in Lewisburg, Pennsylvania. Bats naturally produce antibodies to the fungus, but there is no evidence that these can help them to survive.

Other researchers are promoting a more radical long-term solution: altering airflow in mines where bats hibernate to make the sites less hospitable to the white-nose fungus. Places where bats survive infection tend to be relatively cool and dry. By opening new routes to the outside, researchers could cool and dehumidify the air in mines that are too warm and wet.

There is a chance that manipulating airflow could drive bats to abandon the habitat, says Kate Langwig, an ecologist at the UCSC. But she argues that it is worth a try, because at some sites the white-nose fungus kills about $90 \%$ of the bats present. In the first 5 years that it was present in the United States, the pathogen claimed more than 5.5 million animals.

In the meantime, the United States and Canada are developing and implementing strategies to coordinate work by scientists and by local and national governments - ranging from laboratory and field studies to efforts to prevent people from inadvertently spreading the fungus to pristine caves.

The plight of the bats is "stark - it's demoralizing", says Winifred Frick, an ecologist at the UCSC. "But I have hope in terms of the amount of creative energy and sense of dedication that people are putting forth on this problem. If there are solutions, we will find them."

\title{
First glimpse of primordial stars
}

\section{Astronomers claim to spot generation that seeded Universe.}

\section{BY ELIZABETH GIBNEY}

$\mathrm{S}$ ome of the first generation of stars, whose explosions breathed carbon, oxygen and other elements into the Universe, may have been glimpsed for the first time. The possibility comes as a pleasant surprise to astronomers, who did not expect to be able to spot these primordial objects with existing telescopes.

Primordial stars are theorized to be hundreds of times larger than the Sun, and made up only of pristine hydrogen, helium and traces of lithium left over from the Big Bang. The earliest specimens formed during the first few hundred million years of the Universe, living for only a few million years before exploding in supernovae that laid the seeds for the more element-rich stars to come. But they have never been seen.

A team led by David Sobral, an astronomer at the University of Lisbon, now reports that it may have spied a late-blooming cluster of such stars, in the brightest distant galaxy yet observed. The stars, seen as they were when the Universe was around 800 million years old (a mere $6 \%$ of its current age), seem to be primordial in composition - but, strangely, they also seem to reside in the same galaxy as some second-generation stars.

"Until now, work on these stars has been completely theoretical," says Sobral. "For the first time, we're starting to get observations that can test the many theories about these stars and begin to understand how they formed." His team's report has been posted on the preprint server arXiv and accepted for publication in The Astrophysical Journal (D. Sobral et al. Preprint at http://arxiv.org/ abs/1504.01734; 2015).

Seeing these ancient stars involves observing very distant galaxies. Their light takes billions

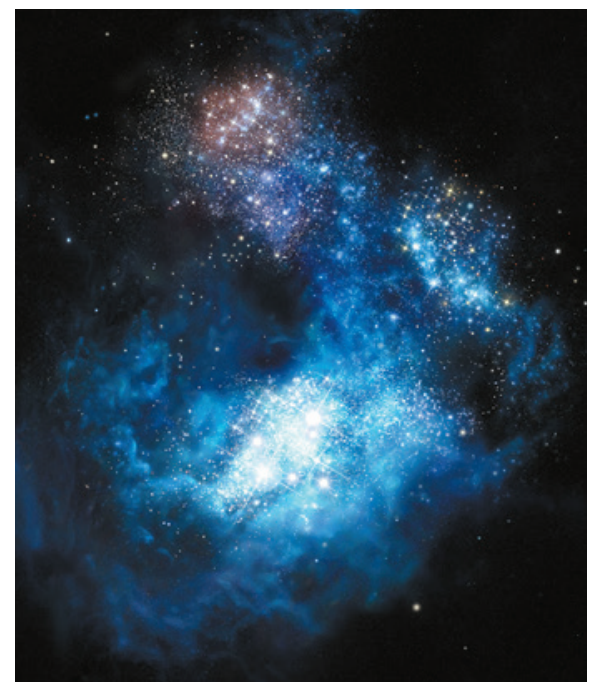

Artist's impression of galaxy CR7.

of years to reach us, and so shows the Universe in its earliest days. But the light is faint, making it difficult to spot. The short lifetimes of the first stars also makes them hard to find.

The surprise discovery emerged after Sobral and an international team of astronomers made a wide sweep of the sky using the Subaru Telescope on Mauna Kea in Hawaii. They used three further telescopes to peer into particularly bright galaxies, and found an intriguing signal from one that they named COSMOS Redshift 7. (The name was chosen so it could be abbreviated as CR7, to echo the nickname of Portuguese footballer Cristiano Ronaldo.)

The spectrum of light from CR7 showed evidence of ionized helium, which suggested that the source of the light was extremely hot. At such temperatures, any carbon and oxygen present should also have ionized, says Sobral. But there was no sign of these elements in

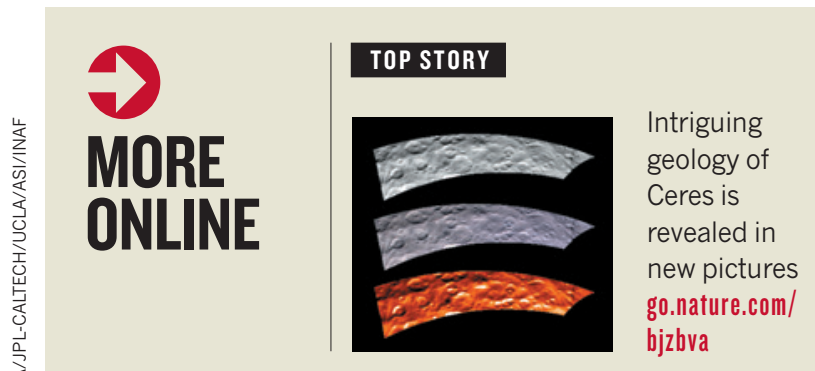

\section{MORE NEWS}

- Nuclear monitoring agency reaches out to scientists go.nature.com/bvtlme - Europe's first humans: what scientists do and don't know go.nature.com/usdonp

- Pope or International Energy Agency — who said what? go.nature.com/as3pr6

\section{NATURE PODCAST}

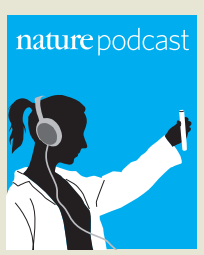

Antarctica's surprising biodiversity - and a new way to diagnose cancer early. nature.com/ nature/podcast 
- the light, which strongly suggested that it came from the first generation of stars.

CR7 also hosts second-generation stars, made from material spat out by dying firstgeneration stars, which means that it is not the sort of galaxy in which astronomers had imagined that they would make such a discovery. Sobral and his colleagues suggest that the primordial stars may be late developers, formed from a cloud of pristine and uncontaminated gas that was prevented from cooling and coalescing by the heat of strong radiation from earlier-blooming stars. This later formation date would also explain why the stars were visible to Subaru.

That primordial stars should turn up in a large and evolved galaxy presents a challenge to the group's interpretation of CR7's light signal, but is probably the least exotic of the possible explanations, says Naoki Yoshida, an astrophysicist at the University of Tokyo. One alternative, that the emission comes from a supermassive black hole that formed directly from the collapse of a pristine cloud of gas, would actually be "even more spectacular", adds Sobral.

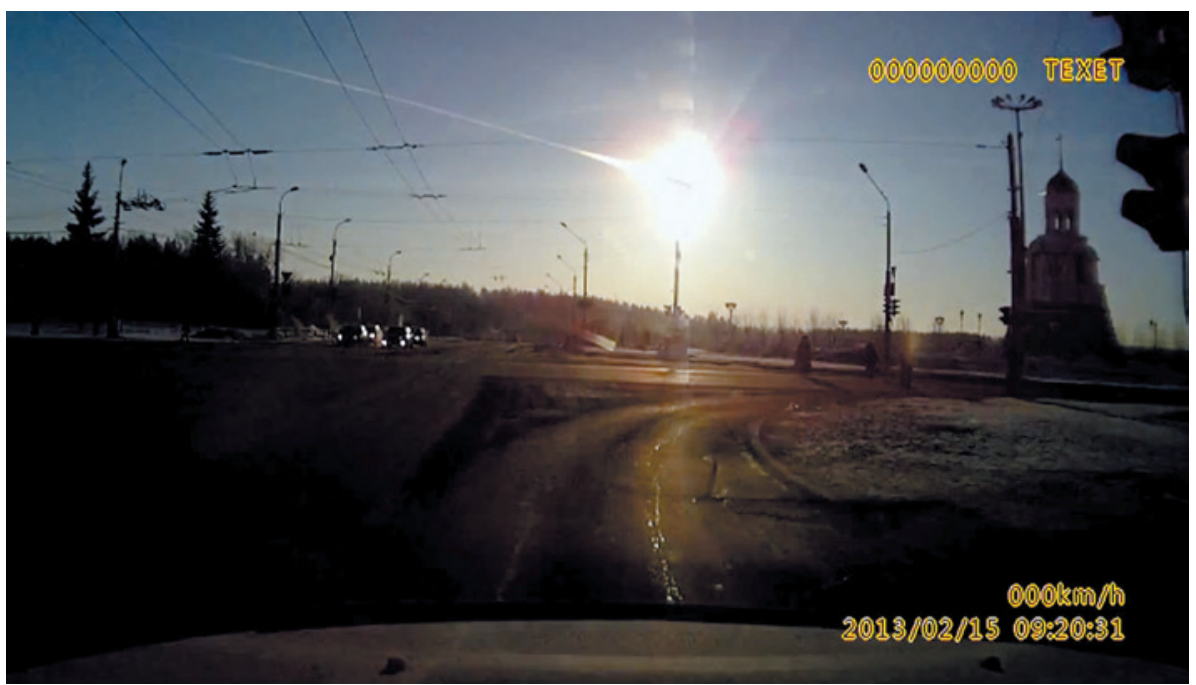

An object estimated at 20 metres across exploded over Chelyabinsk, Russia, in 2013.

\section{PLANETARY SCIENCE}

\section{Private asteroid hunt lacks cash}

\section{Foundation fails to raise funds it needs for a space telescope to catalogue near-Earth objects.}

\section{BY TRACI WATSON}

A stronomy and science organizations have declared 30 June as Asteroid Day, with plans to talk up the danger of asteroids that might be on a collision course with Earth. One partner in the effort, the Sentinel mission, has an especially urgent need to drum up public support: it is struggling to raise the US $\$ 450$ million it needs to launch a space telescope dedicated to finding hundreds of thousands of near-Earth objects.

Astronomers have long sought a spacecraft that would hunt for near-Earth objects full-time, but public funding for such a programme has never materialized. Groundbased surveys have identified nearly all of the largest asteroids, but many of the space rocks that measure between 50 and a few hundred metres in diameter - big enough to wipe out a city with a direct hit - remain uncharted.

"Without space-based assets, progress is going to stall," says physicist Mark Boslough of Sandia National Laboratories in Albuquerque, New Mexico.

Sentinel, announced in 2012 by the B612 Foundation of Mill Valley, California, was supposed to provide that eye in the sky,
Until now, many astronomers believed that seeing the first generation of stars would take an instrument such as NASA's James Webb Space Telescope, which is projected to cost nearly US $\$ 9$ billion, is scheduled to launch in 2018 and should have the power to look further back in time than any previous instrument. But if Sobral and his colleagues are right, it may be possible to see primordial stars with existing telescopes. Indeed, similar bright galaxies have already been identified as potential candidates, adds Sobral. "We may already be looking at them." - and NASA had hoped that the privately run effort would supplement the agency's own asteroid-hunting programme. NASA is considering funding an alternative mission, the Near-Earth Object Camera (NEOCam), initially proposed to the agency in 2006. But some scientists fear that Sentinel could spoil the chances of NASA supporting NEOCam. If Sentinel cannot fly, "then it's just a distraction from getting a job done that needs to get done," says planetary scientist Timothy Swindle of the University of Arizona in Tucson.

Ground-based surveys have located roughly $90 \%$ of the large asteroids that might be a concern - these are all bigger than one kilometre in diameter and pass by Earth's orbit at a distance of 45 million kilometres or less. An impact by one of these behemoths would have global consequences; fortunately, such immense objects plummet to Earth on average only once every 700,000 years or so, and none of those known is heading this way any time soon.

The statistics are less reassuring for smaller objects. As of August 2014, according to data in a recent paper, surveys had spotted only 565 near-Earth asteroids ranging from roughly 45 to 55 metres across - out of an estimated total of up to 520,000 (A. W. Harris and G. D’Abramo Icarus 257, 302-312; 2015). An asteroid no larger than that damaged trees across 2,000 square kilometres of Siberian forest in 1908, in what is known as the Tunguska event (see 'Direct hit'). Tunguska-sized bodies strike Earth once every 500 years or so, Boslough says. An object that exploded over Chelyabinsk, Russia, in February 2013 was estimated to have been about 20 metres in diameter.

\section{PLANETARY PROTECTION}

Sentinel aims to ensure that a similar fate does not unexpectedly befall a place the size of Lagos or London. Mission director Harold Reitsema, who helped to design instruments on the Hubble Space Telescope, says that Sentinel will use an infrared detector to find $90 \%$ of near-Earth objects larger than 140 metres across, and will also spot a good number of objects measuring 30 metres or more. If an asteroid were found to be headed for Earth long enough before impact, authorities could try to knock it off course. 